\title{
GGD 27: X-RAYS FROM A MASSIVE PROTOSTAR WITH AN OUTFLOW
}

\author{
Steven H. Pravdo ${ }^{1}$, Yohko Tsuboi ${ }^{2}$, Yuichiro Suzuki ${ }^{2}$, Timothy J. Thompson ${ }^{3}$, and Luisa Rebull ${ }^{4}$ \\ ${ }^{1}$ Jet Propulsion Laboratory, California Institute of Technology, 306-431, 4800 Oak Grove Drive, Pasadena, CA 91109; spravdo@jpl.nasa.gov \\ ${ }^{2}$ Department of Physics, Faculty of Science and Engineering, Chuo University, Kasuga 1-13-27, Bunkyo-ku, Tokyo 112-8551; Japantsuboi@phys.chuo-u.ac.jp \\ ${ }^{3}$ Jet Propulsion Laboratory, California Institute of Technology, 169-306, 4800 Oak Grove Drive, Pasadena, CA 91109; Timothy.J.Thompson@jpl.nasa.gov \\ ${ }^{4}$ Spitzer Science Center, Caltech M/S 220-6, 1200 E. California Blvd., Pasadena, CA 91125; rebull@ipac.caltech.edu \\ Received 2008 March 17; accepted 2008 September 9; published 2008 December 1
}

\begin{abstract}
We report the discovery of a cluster of Class I protostars in GGD 27. One of these protostars is the previously known, centrally located, GGD 27-ILL, which powers a massive bipolar outflow. We show that GGD 27-ILL, which is known to be the bright infrared (IR) source, IRAS 18162-2048, and a compact radio continuum source, is also the newly discovered hard X-ray source, GGD 27-X. The observations were made with the ACIS instrument on the Chandra X-ray Observatory. The X-rays from GGD 27-X are variable when compared with 4 years earlier, with an unabsorbed 2-10 keV X-ray luminosity in this observation of $1.5-12 \times 10^{31} \mathrm{erg} \mathrm{s}^{-1}$ and a plasma temperature of $\geqslant 10^{7} \mathrm{~K}$. The X-rays are probably associated with the underlying B0 star (rather than outflowing material), providing a rare glimpse in hard X-rays of an optically obscured massive protostar with an outflow. The X-ray luminosity and spectrum appear to be consistent with stars of its type in other star formation regions. Several other variable X-ray sources are also detected in the IR cluster that contains GGD 27-X. We also discuss another nearby cluster. In each of the clusters there is an object that is X-ray hard, highly absorbed at low energies, in a blank optical/IR/radio field, and variable in X-ray intensity by a factor of $\geqslant 10$ on a timescale of 4 years. These latter objects may arise from more recent episodes of star formation or may be "hidden" Class III sources.
\end{abstract}

Key words: infrared: stars - stars: pre-main sequence - X-rays: stars

Online-only material: color figures

\section{INTRODUCTION}

GGD 27 was discovered in a survey to find new Herbig-Haro $(\mathrm{HH})$ objects (Gyulgudaghian et al. 1978) among small, red nebular candidates. While the HH hypothesis for GGD 27 was soon discounted (Hartigan \& Lada 1985), the path of scientific discovery eventually led to its identification as an energy source of HH objects. This scientific path had two interacting branches, one in the radio and the other in the infrared (IR). In the radio Rodríguez et al. (1980) found a water maser and other molecular clouds near GGD 27 that they modeled as mass ejections from an early-B-type star. Rodríguez \& Reipurth (1989) found an elongated $6 \mathrm{~cm}$ radio continuum source in GGD 27 oriented toward the recently discovered $\mathrm{HH}$ objects $\mathrm{HH}$ 80/81 (Reipurth \& Graham 1988). Yamashita et al. (1989) identified a molecular bipolar outflow from GGD 27. Meanwhile, near-IR imaging and polarimetric investigations of GGD 27 found point-like sources (Yamashita et al. 1987) but the illuminating source, GGD 27-ILL, was deemed to be unseen in that band (Aspin et al. 1991). Aspin \& Geballe (1992) concluded from mid-IR spectroscopy that the region surrounding GGD 27-ILL was a pre-main-sequence (PMS) cluster and it is also listed as an IR star cluster (Bica et al. 2003) and the ultracompact H II (UCHII) region, G10.84-2.59 (Kurtz et al. 1994; Thompson et al. 2006). The UCHII region (rather than a much larger ionized region) indicates that the object has not yet reached the main sequence, and has an age $\leqslant 10^{5} \mathrm{yr}$ (Yorke 1986).

Martí et al. (1993) proposed a unifying structure for the GGD 27 complex and associated HH objects based upon their Very Large Array (VLA) observations: it is a high-mass stellar analog of low-mass stars with bipolar outflows, albeit with scales $(\sim 10 \mathrm{pc})$ and luminosities $\geqslant 10$ times larger. $\mathrm{HH} 80 / 81$ is the southern terminus of the outflow and HH 80 North is the northern terminus. The latter is optically invisible due to its location within the molecular cloud (Saito et al. 1999).

At the center of this outflow, at the position of GGD 27, is the source IRAS 18162-2048 (IRAS Point Source Catalog 1985). Rodríguez et al. (1980) calculated a source distance of $1.7 \mathrm{kpc}$ from the associated $\mathrm{CO}$ emission velocity and the Schmidt rotation model (see also Martí et al. 1993). Rodríguez \& Reipurth (1989) concluded from the IRAS luminosity, $\sim 2 \times$ $10^{4} L_{\odot}$ and surrounding ionization, that the source is a PMS B0 star. McCutcheon et al. (1991) assumed the radio continuum emission is thermal and with the IR data determined the central object's effective temperature is $\sim 1.8 \times 10^{4} \mathrm{~K}$. They likewise concluded from its location in the H-R diagram (Panagia 1973) that it is a PMS B0 star.

This power source is associated with VLA source 14 (Martí et al. 1993) = VLA-1 (Gómez et al. 1995). Subsequent highresolution VLA observations (Martí et al. 1995, 1998) showed that condensations within the elongated radio structure moved outward with high proper motions, as expected, corresponding to velocities $\geqslant 500 \mathrm{~km} \mathrm{~s}^{-1}$. They provide a high-precision position for the core of the radio emission, R.A. $=18^{\mathrm{h}} 19^{\mathrm{m}} 12^{\mathrm{s}} .09$ and Decl. $=-20^{\circ} 47^{\prime} 30^{\prime} 9$, which we use throughout as the position of the power source, GGD 27-ILL.

We present the results of a Chandra X-ray Observatory observation of the GGD 27 complex. This is a follow-up to an observation 4 years earlier that was aimed at the southern terminus of the outflow, viz., HH 80/81. The HH objects were detected as luminous X-ray sources and interpreted as the high-temperature shock created by the collision between jet material in the bipolar outflow and the ambient medium (Pravdo et al. 2004, PTM). GGD 27 was also observed, but with the lower spatial resolution of the edge of the field. X-rays were detected in the vicinity of GGD 27-ILL but a higher-resolution 
and higher-sensitivity measurement was required to confirm the identification.

The high positional resolution of observations with the Chandra X-ray Observatory has enabled remarkable advances in the study of X-ray sources from star formation regions. Investigators have identified thousands of X-ray sources in some studies of individual regions and have made significant advances in understanding their X-ray, optical, and IR properties (e.g., Feigelson et al. 2002; Grosso et al. 2005; Teixeira et al. 2006; Wolk et al. 2006; Broos et al. 2007; Prisinzano et al. 2008). The identifications include those of massive stars: O, OB, B, as well as protostellar candidates, with which we will compare the X-rays from GGD 27.

\section{OBSERVATIONS AND ANALYSIS}

\subsection{Chandra X-ray Observatory}

We performed a $72.0 \mathrm{ks}$ Chandra X-ray Observatory (Weisskopf et al. 2002) observation on 2006 May 24-25, with the ACIS-I imaging array (CCDs I0-I3) in the standard mode. Data were reduced using the latest version of the CIAO analysis software.

We performed positional, timing, and spectral analyses of these data. We used a wavelet-based source detection program (Freeman et al. 2002) to locate X-ray sources on CCDs I0-I3 in a soft band, 0.4-2.0 keV, and in a hard band, 2.0-10 keV. We performed encircled energy analysis by examining the spatial distribution of detected sources around their centroids. We constructed light curves to check for source variability.

Finally, we used XSPEC to measure the spectra of the brightest detected sources. Source counts were extracted from regions around sources that matched the local point-spread function (PSF), and backgrounds were taken from nearby source-free regions. The instrumental response matrices are deemed to be reliable at greater than $0.4 \mathrm{keV}$, so we only examined spectra in the range $0.4-10 \mathrm{keV}$. Above $10 \mathrm{keV}$ there were no significant counts. The spectral models included the functions "WABS" for interstellar absorption, and either "MEKA-L" for a thermal spectrum (Mewe et al. 1985) or a power law for the continuum. We calculated the $1 \sigma$ limits for spectral parameters by first allowing all the parameters to vary simultaneously to find the best-fit values. Then a parameter of interest was fixed and we stepped through different values of that parameter, allowing all the other parameters to vary. The value of the interesting parameter when chi squared increased by 3.5 is the $1 \sigma$ limit for a fit with three free parameters (Lampton et al. 1976). We obtained the same results using the C-statistic.

We checked the astrometric accuracy of the X-ray data by comparing the positional output of the source detection program with the positions of unambiguously identified stellar counterparts found in the USNO B1 (Monet et al. 2003) and the Two Micron All Sky Survey (2MASS; Skrutskie et al. 2006) catalogs. For the $15 \mathrm{X}$-ray sources that had positional coincidence of less than 0.'5 with USNO B1 objects, the average and standard deviations of the right ascension and declination offsets were $-0.09 \pm 0$.'16 and $0.04 \pm 0.24$, respectively.

The possibility exists that extragalactic X-ray sources penetrate the Saito cloud and are detected in our observation. Feigelson et al. (2002) estimated that in a similar observation $\leqslant 2 \%$ of the sources are extragalactic contaminants. This implies that less than one of our sources is from the extragalactic background.

\subsection{Spitzer Space Telescope}

We also make use of Spitzer Space Telescope (Werner et al. 2004) archived data from the Infrared Array Camera (IRAC, Fazio et al. 2004) and the Multiband Imaging Photometer for Spitzer (MIPS, Rieke et al. 2004). The observation was performed on 2005 September 15. We created deconvolved images and outlier masks with the Spitzer Science Center (SSC) Mosaicking and point-source extraction (MOPEX) software package (Makovoz \& Marleau 2005). We applied the outlier masks, along with data collection event and permanent bad pixel masks from the SSC pipeline. The sources are oversampled with 0.25 arcsec pixels to match the deconvolved images.

For IRAC we created enhanced-resolution images in the four bands-3.6, 4.5, 5.8, and $8.0 \mu \mathrm{m}$-using the HiRES software package developed at JPL (Backus et al. 2005), and IRAC point-response functions (PRFs) distributed through an SSC-contributed software package, "Iracworks" . Resolution is enhanced by a factor of $\sim 3$ with HiRES software (Velusamy et al. 2007) and the algorithm properly conserves flux (Backus et al. 2005). Although this results in ringing around bright point sources with extended background emission, it does concentrate the flux in the Airy rings for point sources, and better defines the shape of extended sources, and thus is best for deblending crowded sources, or distinguishing the flux of a point source from the background around it.

We performed source extraction on the images using the SExtractor software (Bertin \& Amouts 1996). This returns an integrated flux for each source in each image. We calculated both an aperture flux within a fixed circular aperture tuned to the known PSF (which we report herein) and an isophotal flux (for a check). We also examined the elongations of the images since a point-like source would have an elongation close to 1 .

To verify our IRAC astrometry we compared these objects with coincident X-ray sources within 0.5. For 28 sources in IRAC $3.6 \mu \mathrm{m}$ band, the offsets were $0.08 \pm 0.21$ in R.A. and $0.06 \pm 0$ ' 18 in Decl. Comparing X-ray sources with IRAC $8.0 \mu \mathrm{m}$ sources yields offsets of $0.03 \pm 0.20$ in R.A. and $0.12 \pm 0.27$ for 11 sources.

Most of the MIPS data were unusable because of strong saturation from GGD 27. In the northeast region we were able to extract some source information but because there were so few isolated sources the astrometry could only be checked via registration by eye and appears to be accurate to better than $1^{\prime \prime}$ after the fact.

\section{RESULTS}

This is a star formation region and thus contains many $\mathrm{X}$-ray bright objects. Many of these were detected in the earlier observation (PTM). In this work we concentrate on X-rays from the GGD 27 region and the MRR 32 region (Martí et al. 1993) to the northeast.

\subsection{X-rays Sources in GGD 27 IR Cluster}

Figure 1 shows the Chandra/ACIS X-ray image of the GGD 27 IR cluster (large circle), which has a $\sim 1.5$ diameter (Bica et al. 2003). There were 16 X-ray sources detected within the cluster by the automatic processing. These are labeled in the figure. Further examination showed that three of the sources-6, 9 , and 12-were close doubles, each pair called " $a$ " and " $b$ ". The intensity of sources 2-5 may be affected by the lower efficiency

\footnotetext{
5 http://ssc.spitzer.caltech.edu/archanaly/contributed/browse.html.
} 


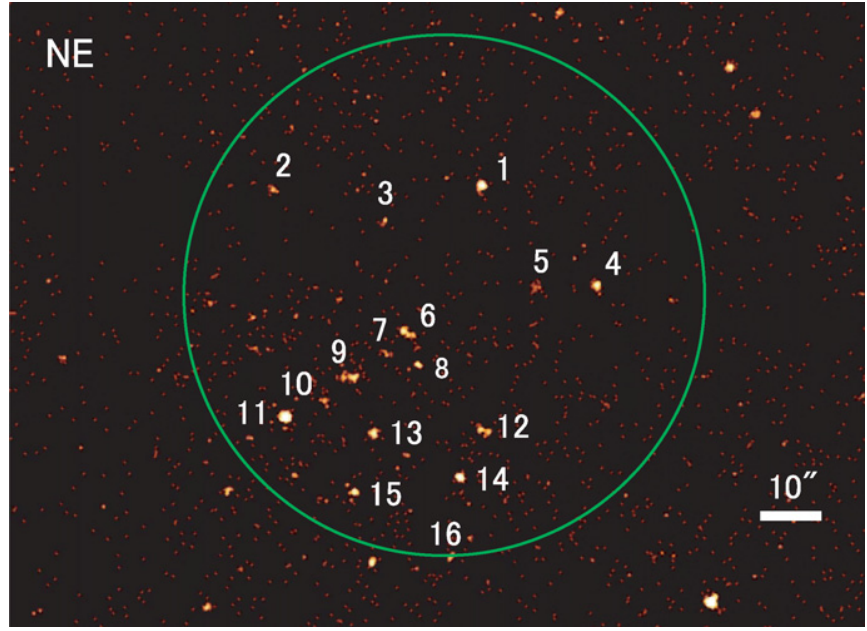

Figure 1. Chandra/ACIS broadband X-ray (0.4-10 keV) image of the GGD 27 IR cluster (circle). X-ray sources detected with the automatic processing software are numbered.

in the gap between chips. (The efficiency is nonzero because of the standard Chandra pointing dither.)

Figure 2 shows Gaussian-smoothed images of the X-rays in soft and hard bands around the region of GGD 27-ILL and the Spitzer/IRAC data in two bands. The X-ray sources detected in all bands (circles) are also shown.

We cross-correlated the X-ray sources with sources from the USNO B1 and 2MASS catalogs and those we detected in the Spitzer/IRAC data. The Spitzer/MIPS data were saturated in this region. The results are in Table 1. Six of the 19 X-ray sources are in blank optical/IR fields. The others are detected in all or some of the optical, near-IR, and mid-IR bands. We constructed spectral energy distributions (SEDs) for these. The stars are divided into young stellar object (YSO) classes by computing their SED indices according to the classification scheme described by Greene et al. (1994). We find four Class I
(Figure 3(a)), three Class II (Figure 3(b)), and five Class III stars (Figure 3(c)). See also Table 1 for details. Source 7 has an optical but no IR counterparts and is not classified (see Section 4.1). The Class I stars are denoted by cyan circles in Figure 2. They are all hard-X-ray-only sources except for source 15 and are located within $\sim 40^{\prime \prime}$ of GGD 27-X (9a) which is itself a Class I protostar (McCutcheon et al 1991).

We determined the observed X-ray luminosities (Column 4) for each source by converting counts determined from the wavelet-based source detection in the soft and hard bands (Column 3) to intensities, adding, and assuming a distance of $1.7 \mathrm{kpc}$. For the brightest sources with the most counts we also fit the spectra. This gives a more accurate estimate of the intensity and allows us to calculate an "unabsorbed" luminosity, assuming the low-energy absorption is between the source and us. These values when calculated are shown in parenthesis below the values with absorption (also Column 4).

\subsection{X-ray Variability in the GGD 27 Cluster}

There is evidence of flaring activity (factors of a few, timescales of tens of thousands of seconds) in some of the brighter sources during the course of the 2006 observation (see Section 4.3). There is also significant variability when we compare these data to those of PTM. Figure 4 shows the region of the GGD 27 IR Cluster observed with Chandra in 2002 (PTM), Gaussian-smoothed in the $0.4-10 \mathrm{keV}$ band. The most significant observational difference was that in 2002 GGD 27 was $\sim 5^{\prime}$ off-axis resulting in a PSF that is both significantly degraded in size and asymmetric. At $3.5 \mathrm{keV}$ the $70 \%$ encircled energy radius is $\sim 5^{\prime \prime}$. At higher energies the radii get even larger. ${ }^{6}$ This is in contrast to the on-axis PSF that is $\sim 1^{\prime \prime}$ for $70 \%$ encircled energy. Nevertheless the degradation in the PSF is not expected to significantly displace the centroid of the emission. Therefore, we can compare the relative intensities across the complex in the two observations shown in Figures 1 and 4.

\footnotetext{
6 http://cxc.harvard.edu/cal/Acis/Cal_prods/psf/eer_off.html.
}
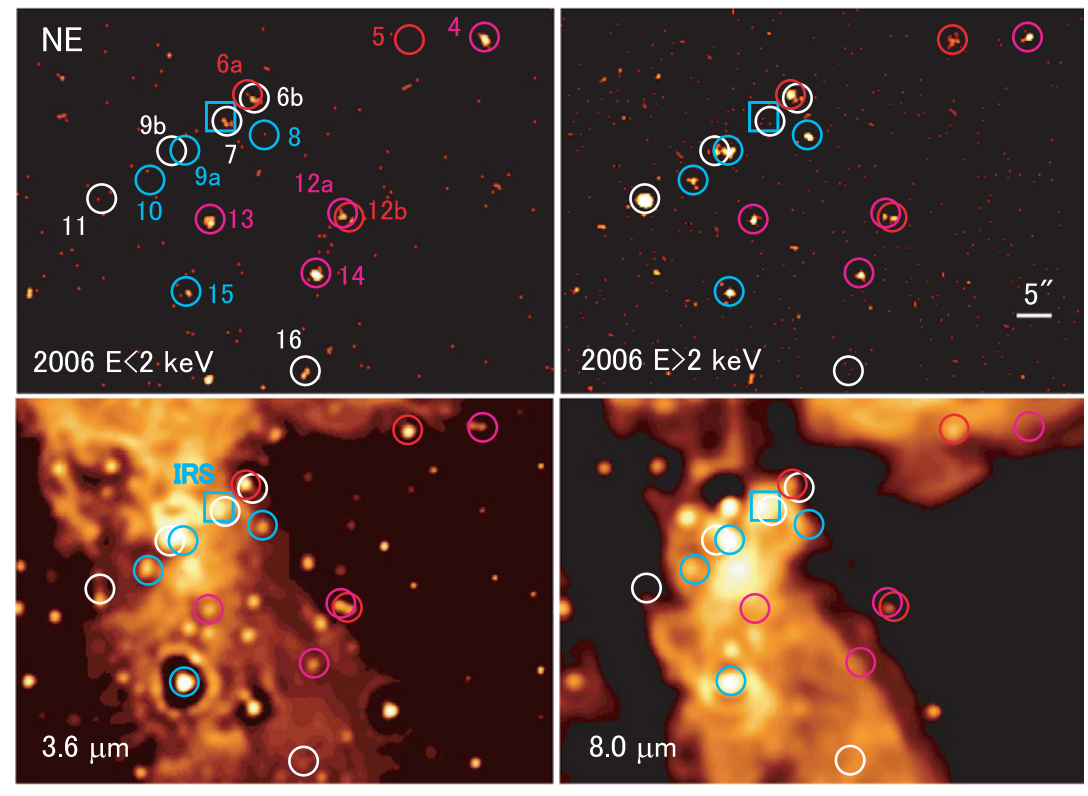

Figure 2 Chandra/ACIS X-ray and Spitzer/IRAC IR images of the GGD 27 cluster. The X-ray images are in a soft, 0.4-2 keV, and a hard band, 2.0-10 keV, and have been Gaussian-smoothed. The IR images are from IRAC bands 1 and 4. The cyan (Class I), red (Class II), magenta (Class III), and white (unclassified) 2" radius circles depict the positions of X-ray sources detected at all X-ray energies with the wavelet source detection software and by human inspection for close "doubles". The cyan square is the Class I source "IRS" that was not detected in X-rays. 
Table 1

X-ray Sources in GGD 27 IR Cluster

\begin{tabular}{|c|c|c|c|c|c|c|c|c|c|c|c|c|}
\hline No. & R.A. (2000) & Decl. & $0.4-2 \mathrm{keV}$ & $2-10 \mathrm{keV}$ counts ks ${ }^{-1}$ & $\begin{array}{c}2006 L_{\mathrm{x}}^{\mathrm{a}} \\
10^{30} \mathrm{erg} \mathrm{cm}^{-2} \mathrm{~s}^{-1}\end{array}$ & $\begin{array}{c}2002 L_{\mathrm{x}}^{\mathrm{a}} \\
10^{30} \mathrm{erg} \mathrm{cm}^{-2} \mathrm{~s}^{-1}\end{array}$ & Other Names ${ }^{\mathrm{b}}$ & 2MASS & $\begin{array}{c}\text { Spitzer } \\
\text { IRAC Bands }\end{array}$ & $\begin{array}{l}2-10 \mu \mathrm{m} \\
\text { Index }^{\mathrm{c}}\end{array}$ & $\begin{array}{c}\text { IR } \\
\text { Spec. Class }\end{array}$ & $\overline{\text { References }}$ \\
\hline 1 & 274.79386 & -20.78280 & $0.42 \pm 0.08$ & $0.28 \pm 0.06$ & $\begin{array}{c}4.9 \\
(5.1)\end{array}$ & $5.2-18$ & $\begin{array}{c}\text { HL 41, MRR 12, CXOPTM } \\
\text { J181910.4-204657 }\end{array}$ & $18191051-2046578$ & $1-4$ & -1.3 & III & $1,2,3$ \\
\hline 2 & 274.80462 & -20.78300 & $\ldots$ & $0.10 \pm 0.04$ & 1.5 & .. & Empty OIR field & $\ldots$ & $\ldots$ & & $\ldots$ & $\ldots$ \\
\hline 3 & 274.79892 & -20.78455 & $\cdots$ & $0.09 \pm 0.04$ & 1.3 & 20 & $\begin{array}{l}\text { Empty OIR field } \\
\text { CXOPTM } \\
\text { J181912.0-204703 }\end{array}$ & $\cdots$ & $\cdots$ & & $\cdots$ & $\cdots$ \\
\hline 4 & 274.78794 & -20.78757 & $0.23 \pm 0.06$ & $0.17 \pm 0.05$ & 3.0 & 0.34 & $\begin{array}{c}\text { CXOPTM } \\
\text { J181909.1-204716 }\end{array}$ & 18190910-2047149 & $\cdots$ & -1.9 & III & 3 \\
\hline 5 & 274.79108 & -20.78767 & $\ldots$ & $0.15 \pm 0.05$ & 2.3 & $\ldots$ & & 18190984-2047155 & $1-3$ & 0.09 & II & $\ldots$ \\
\hline $6 a$ & 274.79786 & -20.78978 & $\ldots$ & $0.26 \pm 0.06$ & 3.9 & $\ldots$ & IRS 9 (NE source) & 18191146-2047229 & $1-4$ & 0.31 & II & 4 \\
\hline $6 \mathrm{~b}$ & 274.79756 & -20.78996 & $0.12 \pm 0.04$ & $\ldots$ & 0.22 & $\cdots$ & $\begin{array}{l}\text { Empty OIR field } \\
\text { (SW source) }\end{array}$ & $\cdots$ & $\cdots$ & & $\cdots$ & $\cdots$ \\
\hline 7 & 274.79871 & -20.79086 & $0.08 \pm 0.03$ & .. & 0.15 & 0.23 & HL 34, CS 1 & $\ldots$ & $\ldots$ & & $\cdots$ & 1,5 \\
\hline 8 & 274.79719 & -20.79142 & $\cdots$ & $0.19 \pm 0.05$ & 2.9 & $\ldots$ & $\cdots$ & $\cdots$ & $1-3$ & 2.5 & I & $\cdots$ \\
\hline $9 \mathrm{a}$ & 274.80047 & -20.79200 & $\cdots$ & $0.31 \pm 0.07$ & $\begin{array}{c}4.6 \\
(15-120)\end{array}$ & $\cdots$ & $\begin{array}{l}\text { GGD 27-ILL, MRR } 14 \\
\text { IRAS 18162-2048 } \\
\text { GGD 27-X }\end{array}$ & $\cdots$ & $3-4$ & 2.6 & I & $2,6,7$ \\
\hline $9 b$ & 274.80102 & -20.79202 & $\cdots$ & $0.13 \pm 0.04$ & 1.9 & $\cdots$ & $\begin{array}{l}\text { Empty optical/IR } \\
\text { field (E source) }\end{array}$ & $\cdots$ & $\cdots$ & & $\cdots$ & $\cdots$ \\
\hline 10 & 274.80193 & -20.79316 & $\cdots$ & $0.12 \pm 0.04$ & 1.8 & 16 & $\begin{array}{c}\text { CXOPTM } \\
\text { J181912.0-204703 }\end{array}$ & $\cdots$ & $1-4$ & 4.0 & I & 3 \\
\hline 11 & 274.80395 & -20.79389 & $\cdots$ & $1.83 \pm 0.16$ & $\begin{array}{l}17 \\
(100)\end{array}$ & $\cdots$ & Empty OIR field & $\cdots$ & $\cdots$ & & $\cdots$ & $\cdots$ \\
\hline $12 \mathrm{a}$ & 274.79387 & -20.79455 & $0.12 \pm 0.04$ & $\cdots$ & 0.22 & $\cdots$ & (NE source) & $\cdots$ & $1-3$ & -0.90 & III & $\cdots$ \\
\hline $12 b$ & 274.79360 & -20.79461 & $\cdots$ & $0.09 \pm 0.04$ & 1.3 & $\ldots$ & (SW source) & 18191048-2047402 & $1-4$ & -0.37 & II & $\cdots$ \\
\hline 13 & 274.79941 & -20.79469 & $0.19 \pm 0.05$ & $0.18 \pm 0.05$ & 3.0 & 3.8 & $\begin{array}{c}\text { CXOPTM } \\
\text { J181911.8-204740 }\end{array}$ & 18191184-2047406 & $1-2$ & -1.4 & III & 3 \\
\hline 14 & 274.79495 & -20.79683 & $\cdots$ & $0.16 \pm 0.05$ & 2.3 & 1.2 & $\begin{array}{c}\text { CXOPTM } \\
\text { J181910.8-204748 }\end{array}$ & 18191078-2047481 & $1-2$ & -1.5 & III & 3 \\
\hline 15 & 274.80042 & -20.79752 & $0.26 \pm 0.06$ & $0.24 \pm 0.06$ & $\begin{array}{l}2.6 \\
(4)\end{array}$ & $\cdots$ & IRS 1 & 18191208-2047508 & $1-4$ & 0.94 & I & 1,8 \\
\hline 16 & 274.79543 & -20.80064 & $0.09 \pm 0.04$ & $\cdots$ & 0.17 & $\cdots$ & Empty OIR field & $\cdots$ & $\ldots$ & & $\cdots$ & $\cdots$ \\
\hline
\end{tabular}

Notes.

${ }^{a}$ Values in parentheses are unabsorbed luminosities when measured in the $0.4-10 \mathrm{keV}$ band.

${ }^{\mathrm{b}}$ OIR $=$ optical/IR

${ }^{c}$ SED slope as in Greene et al. (1994).

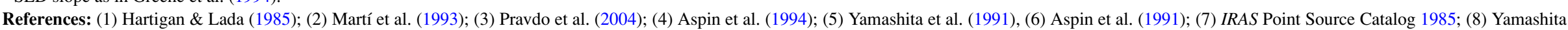
et al. (1987). 




(a)

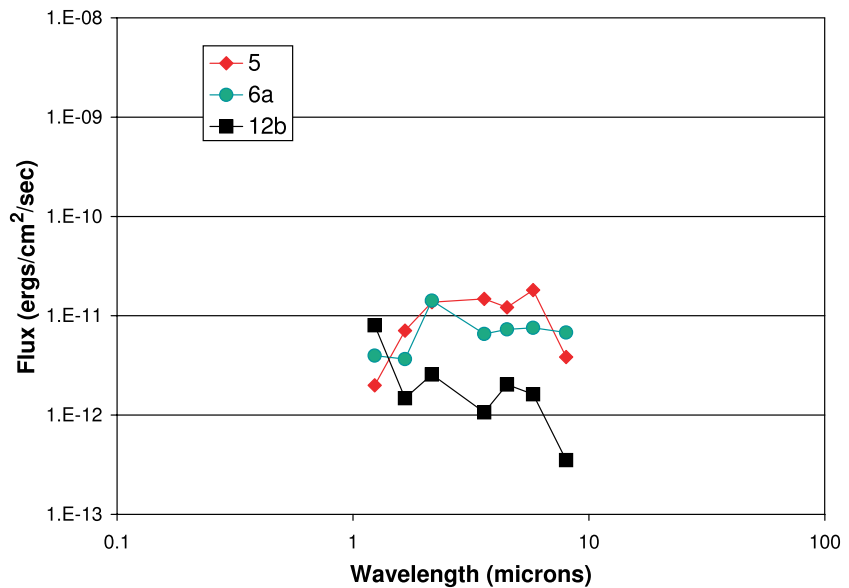

(b)

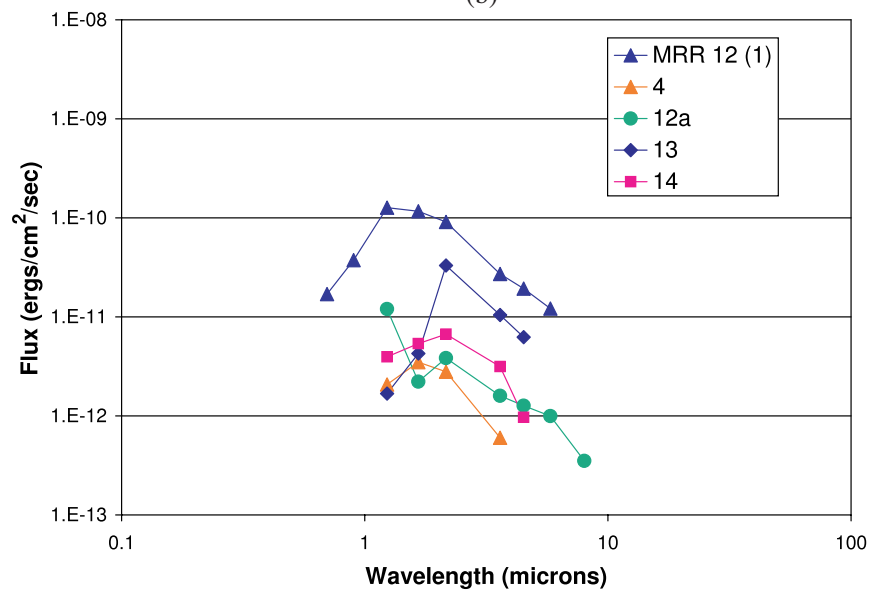

(c)

Figure 3. Spectral energy distributions (SEDs) of the optical/IR counterparts of the X-ray sources in the GGD 27 IR cluster: (a) Class I, (b) Class II, and (c) Class III sources.

All the sources detected in 2002 are also seen in 2006 with the possible exception of source 3 (offset by a couple of arcsec from its PTM counterpart). Table 1 lists the luminosities in both epochs. Sources 10 (see the following Section) and 3 show the largest dimming by factors $\sim 10$. The former is a Class I protostar while the latter is in a "blank field" dominated by visible-IR nebular emission. Source 4, a Class III PMS, is brightened by about the same factor. The other four sources were variable at no more than a factor of $\sim 2$. Twelve other sources seen in 2006 were not detectable in 2002, including the

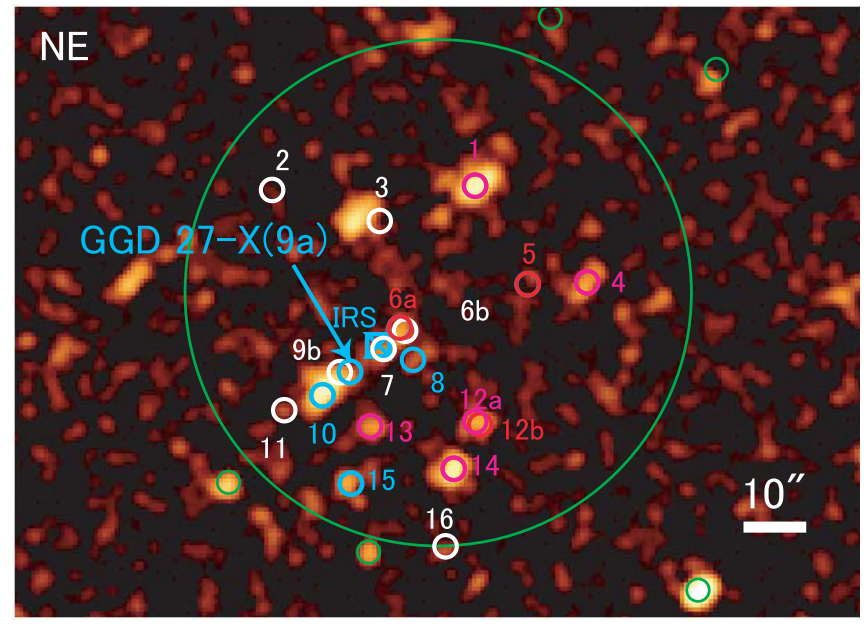

Figure 4. Gaussian-smoothed Chandra/ACIS images of the GGD 27-X region from 2002 in the $0.4-10 \mathrm{keV}$ band. The small ( $2^{\prime \prime}$ radius) circles are the locations of ACIS X-ray sources detected with the standard processing in 2006 with the same color coding as in Figure 2. The large circle shows the extent of the GGD 27 IR cluster, and the green circles show out-of-cluster X-ray sources.

brightest X-ray source 11, also in a blank field. We use a "blank field" in what follows to mean there is no optical, IR, or radio counterpart.

\section{3. $G G D 27-X=G G D$ 27-ILL}

The X-ray source CXOPTM J181912.4-204733 is centered about 4 " southeast of GGD 27-ILL. It was the brightest source in the vicinity of GGD 27-ILL (Figure 4) in 2002 (PTM). This source is detected again in 2006 but is significantly dimmer-source 10 in Table 1. It is now clear that this source is not GGD 27-ILL and is instead one of the several nearby, surrounding, strong X-ray emitters associated with a different Class I protostar.

The newly discovered X-ray source 9a is coincident with GGD 27-ILL at the X-ray resolution limit, $\sim 0$.5. In what follows we refer to source 9a as GGD 27-X. Figure 5 shows the location of GGD 27-X (0'.5 radius circle) superimposed upon the IRAC images (linear scale) in each of the four bands. It is evident that an IR source to the northeast of GGD 27-X fades with increasing wavelength while GGD 27-ILL = GGD27-X brightens. The IR source to the northeast is IRS2 (Yamashita et al. 1987). It has no associated X-rays, and is not a stellar object, rather arising from reflected light (Aspin et al 1991, Stecklum et al. 1997). Figure 5 also shows the position of the center of the radio outflow (0'.25 green square; Martí et al. 1998), within 0.5 of the mid-IR and X-ray emissions.

GGD $27-X$ is part of a close double with source $9 \mathrm{~b}, 2^{\prime \prime}$ east (Figure 6). The number of X-ray counts from sources 9a (GGD 27-X) and $9 \mathrm{~b}$ are 20 and 11, respectively. Neither of the objects show X-rays with $E<2 \mathrm{keV}$, indicative of hard spectra with low-energy absorption. The preponderance of $E>5 \mathrm{keV}$ $\mathrm{X}$-rays arise from GGD 27-X. Spectral fitting of the GGD 27-X spectrum using a thermal model with standard abundances and low-energy absorption indicates a temperature $T \geqslant 10^{7} \mathrm{~K}$ (best fit of $10^{8} \mathrm{~K}$ ) with low-energy absorption, $N_{H} \geqslant 7 \times 10^{22} \mathrm{H}$ $\mathrm{cm}^{-2}$ (best fit of $2.4 \times 10^{23} \mathrm{H} \mathrm{cm}^{-2}$ ), at $1 \sigma$ confidence using either the chi squared or C-statistic.

We searched for diffuse X-ray emission in the vicinity of GGD 27-X. Figure 7 shows the radial profiles of the X-ray emission from both observations centered at GGD 27-X and 

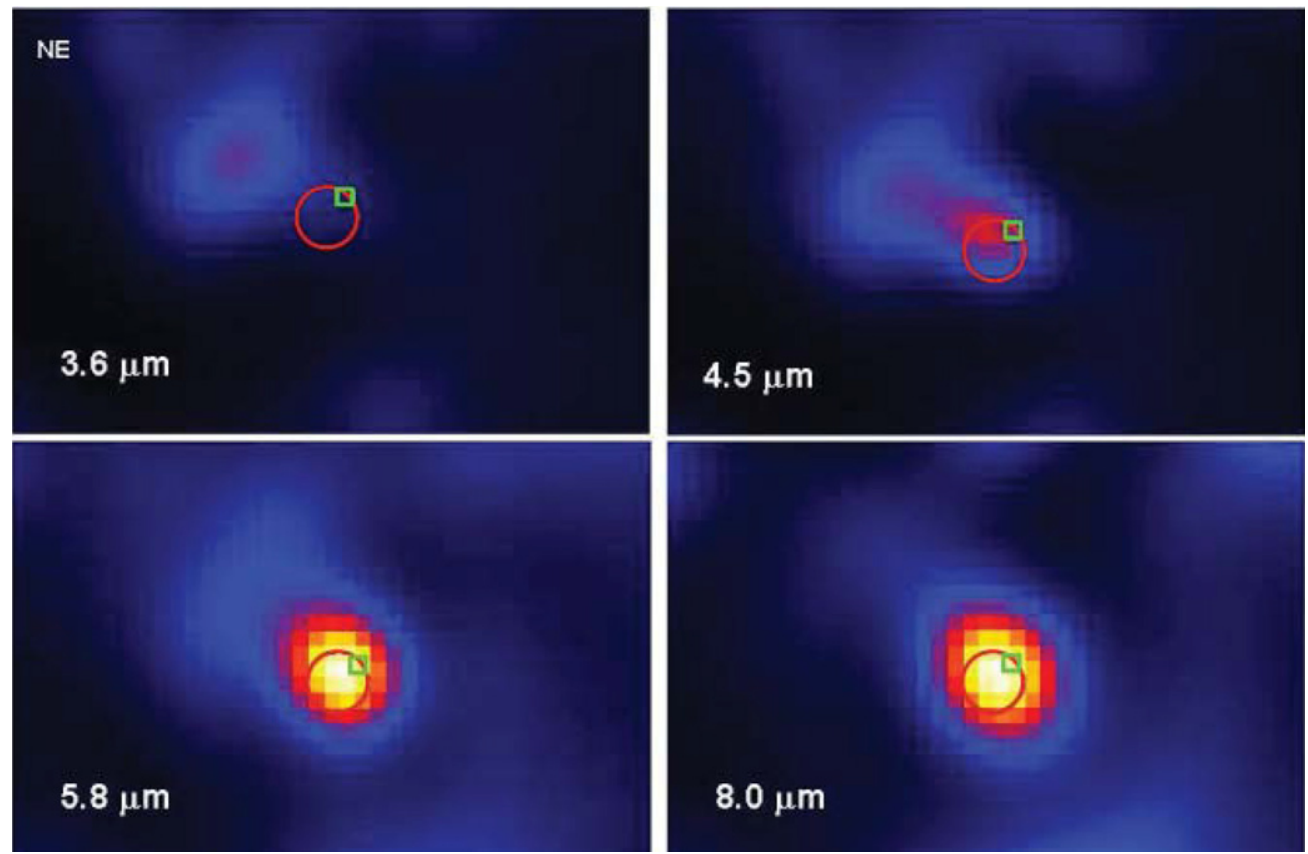

\section{$4.5 \mu \mathrm{m}$}



Figure 5. The IRAC images (linear intensity scale) of the GGD 27-ILL region in the four bands. The position of GGD 27-X (red circle, 0'.5 radius) is shown. Also shown is the center of the radio outflow (green square; $0{ }^{\prime \prime} 25$ sides).

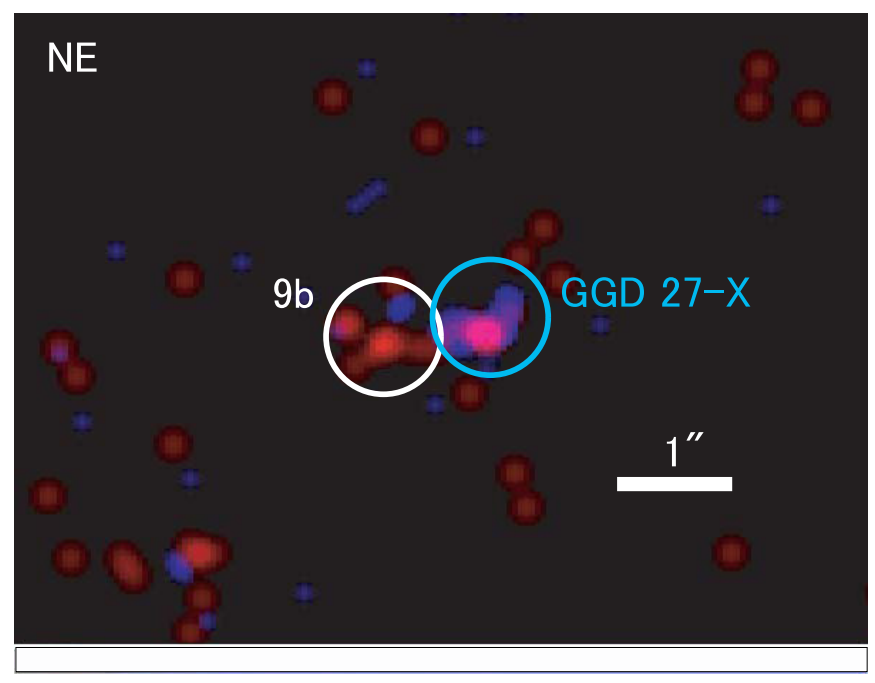

Figure 6. X-ray image of GGD 27-X. Counts with $2<E<5 \mathrm{keV}$ are shown in red. Counts with $5<E<8 \mathrm{keV}$ are shown in blue. The circles have $1^{\prime \prime}$ radius.

in annuli that match the resolution of each observation. Since the Chandra PSF changes as a function of off-axis angle (see Section 3.2), and the off-axis angle itself changes between the two observations, these plots are a convolution of this changing PSF with the actual X-ray distribution. Nevertheless, we determined that within the noise limits, the emission is dominated by individual sources. For example, the peak at $\sim 10^{\prime \prime}$ radius in 2006 is due to source 11 .

\subsection{Other X-ray Sources in the GGD 27 Cluster}

The brightest $\mathrm{X}$-ray source in the cluster is source 11. It was not detected in 2002 with an upper limit of $\sim 0.1 \mathrm{cts} \mathrm{ks}^{-1}$ in the hard band, and thus brightened by a factor greater than 10 in the intervening time. There is no optical/IR counterpart and thus no indication of the source class except for the X-ray evidence. The

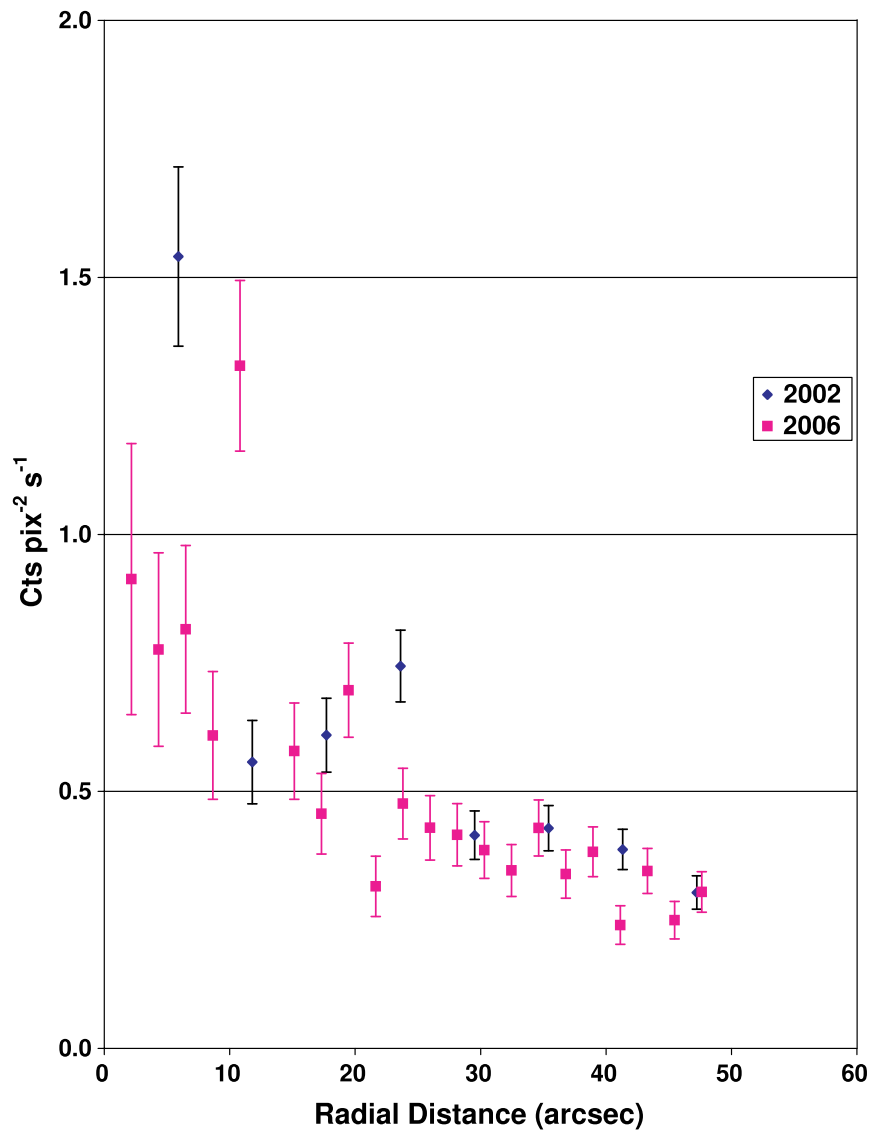

Figure $7 \mathrm{X}$-ray surface brightness in annular regions around GGD 27-X from the observations in 2002 and 2006 in the $2-10 \mathrm{keV}$ band.

thermal model fit indicates $T=1.5(+1.7,-0.5) \times 10^{7} \mathrm{~K}$ and $N_{H}=2.3(+0.7,-1.1) \times 10^{23} \mathrm{H} \mathrm{cm}^{-2}$. Another five of the sources are in blank fields. Two of these are soft X-ray-only sources $(E<2 \mathrm{keV})$. 

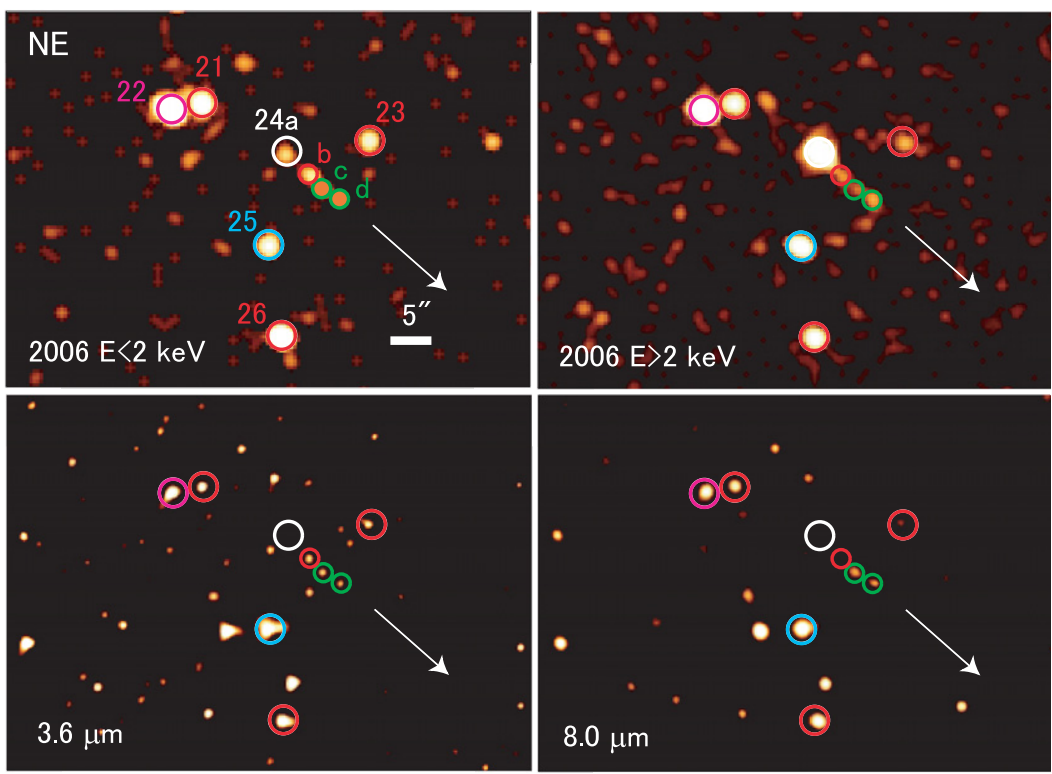

Figure 8 Chandra/ACIS and Spitzer/IRAC images of the MRR $32 \mathrm{X}$-ray cluster $\sim 3^{\prime}$ northeast of GGD 27. The symbols have the same meaning as in Figure 2 with the addition of green circles depicting "flat" sources. The arrow shows the direction toward GGD 27-ILL

Sources 6a and 15 correspond to IRS 9 and 1, respectively, described by Stecklum et al. (1997), although they only detected IRS 1 in the mid-IR. Here we identify them as X-ray-emitting Class II (source 6a) and Class I (source 15) protostars. We extracted a spectrum for source 15 to determine to first order its low energy absorption. We find $N_{H} \geqslant 1 \times 10^{22} \mathrm{H} \mathrm{cm}^{-2}$.

The only X-ray sources with optical counterparts are sources 1 and 7. Source 1 is also an IR/radio continuum source (Martí et al. 1993). PTM discuss the high relative radio flux in this object and the possibility of its nonthermal origin. It now appears to be confirmed as a Class III PMS star based on its IR SED. Source 7 is discussed further in Section 4.1.

\subsection{The MRR 32 Cluster}

Another small cluster of X-ray sources lies $\sim 3^{\prime}$ northeast of GGD 27-X (Figure 8). The X-ray source CXOPTM J181921.8204535 (PTM) is redetected and found to be a double-sources 21 and 22-the latter being MRR 32 (Martí et al. 1993) = HL 8 (Hartigan \& Lada 1985). It is the brightest local X-ray source and among the brightest in the IR. A total of six X-ray sources were detected by the standard software and are shown in the figure. Only one other X-ray source was previously detected by PTM, CXOPTM J 181920.9-204609, source 26. Source 24a is the only X-ray source in a blank field. A distribution of dimmer $\mathrm{X}$-ray sources, sources $24 \mathrm{~b}-\mathrm{d}$, a "string of pearls," appears in line with source 24a. These were below the threshold for automatic detection but we manually determined their X-ray intensities because their obvious presence in the Spitzer data confirms their existence. The sources in this string have varying X-ray hardness and IR reddening, probably indicative of higher column density as their depth in the cloud varies relative to the line of sight. The morphology may also be the result of triggered star formation. The source properties from this cluster are reported in Table 2.

Sources 24a (see Section 4.3) and 26 show flaring behavior typical of some PMS stars. The long-term variability between the 2002 and 2006 epochs for sources 22 (MRR 32) and 26 is at most $\sim 2$. The combined intensities from sources 21 and 22 are close to the unresolved 2002 measurement that probably contained both objects. Source 24a must have brightened by a factor $\geqslant 6$ to not have been detected in 2002 .

The bright blank-field source 24a has a hard, absorbed X-ray spectrum with $T>4 \times 10^{7} \mathrm{~K}$ and $N_{H}=7.2(+2.8,-1.7) \times$ $10^{22} \mathrm{H} \mathrm{cm}^{-2}$. In contrast two other bright and/or variable sources are neither as hot nor as absorbed. MRR 32 has $T=2.5$ $(+0.6,-0.5) \times 10^{7} \mathrm{~K}$ and $N_{H}=(1.0 \pm 0.2) \times 10^{22} \mathrm{H} \mathrm{cm}^{-2}$ and source 26 has $T=2.8(+2.2,-1.3) \times 10^{7} \mathrm{~K}$ and $N_{H}=$ $0.3(+1.1,-0.2) \times 10^{22} \mathrm{H} \mathrm{cm}^{-2}$.

We also found optical/2MASS counterparts to the X-ray sources and measured their IRAC intensities. Due to the separation of this region from the saturated environs of GGD 27, MIPS data were not saturated and we were able to measure the $24 \mu \mathrm{m}$ fluxes from the sources. Table 2 also contains the IR classes determined from the SEDs shown in Figures 9(a) and 9(b). The line-of-sight absorption for the $24 \mathrm{a}-\mathrm{d}$ string varies from object to object. Source 24a is the most absorbed: it has no counterpart at other wavelengths and high soft-X-ray absorption. Source $24 \mathrm{~b}$ is the least absorbed as it features the highest ratio of soft to hard X-rays and a 2MASS counterpart. Sources 24c and $24 \mathrm{~d}$ are intermediate in X-ray absorption and have no 2MASS counterparts. However, the latter two have flat SEDs based upon their Spitzer (mid-IR) counterparts.

\section{DISCUSSION}

\subsection{A Class I Protostar Cluster}

The region within $0.1 \mathrm{pc}$ of GGD 27-X is a cluster containing at least four Class I protostars. GGD 27-X is still the only identifiable high-mass star among the group. As a group the Class I X-ray sources exhibit $\geqslant 10$ variability between the two epochs. Interestingly the object IRS 7, that Stecklum et al. 1997 concluded was a stellar source, was not detected in X-rays in either observation.

The compact distribution of the Class I objects (Figure 2) might be due to chance superposition but could also illustrate the effect of triggered star formation (see Sugitani et al. 1995) over timescales of $\sim 10^{5}-10^{6} \mathrm{yrs}$. In this scenario, GGD 27-ILL triggered star formation in a northwest line containing many of the 
Table 2

X-ray Sources in MRR 32 Region

\begin{tabular}{|c|c|c|c|c|c|c|c|c|c|c|c|c|}
\hline No. & R.A.(2000) & Decl. & $0.4-2 \mathrm{keV}$ & $2-10 \mathrm{keV} \mathrm{cts} \mathrm{ks}^{-1}$ & $\begin{array}{c}2006 L_{\mathrm{x}}^{\mathrm{a}} \\
10^{30} \mathrm{erg} \mathrm{cm}^{-2} \mathrm{~s}^{-1}\end{array}$ & $\begin{array}{c}2002 L_{\mathrm{x}}^{\mathrm{a}} \\
10^{30} \mathrm{erg} \mathrm{cm}^{-2} \mathrm{~s}^{-1}\end{array}$ & Other Names & 2MASS & $\begin{array}{c}\text { Spitzer } \\
\text { IRAC } \\
\text { MIPS Bands }\end{array}$ & $2-10 \mu \mathrm{m}$ Index ${ }^{\mathrm{c}}$ & $\begin{array}{c}\text { IR } \\
\text { Spec. Class }\end{array}$ & $\overline{\text { References }}$ \\
\hline 21 & 274.84078 & -20.76005 & $0.72 \pm 0.10$ & $0.47 \pm 0.08$ & 8.5 & $46-50^{\mathrm{b}}$ & $\ldots$ & $18192177-2045359$ & $\begin{array}{c}1-4 \\
+ \text { MIPS } 24\end{array}$ & -0.41 & II & $\ldots$ \\
\hline 22 & 274.84208 & -20.76030 & $5.33 \pm 0.27$ & $3.15 \pm 0.21$ & $\begin{array}{c}29 \\
(70)\end{array}$ & $46-50^{\mathrm{b}}$ & $\begin{array}{c}\text { HL 8, MRR 32 } \\
\text { CXOPTM J181921.8-204535 }\end{array}$ & $18192208-2045368$ & $1-4$ & 0.22 & III & $1,2,3$ \\
\hline 23 & 274.83359 & -20.76156 & $0.23 \pm 0.06$ & $0.06 \pm 0.03$ & 1.3 & $\ldots$ & $\begin{array}{l}0692-0595850 \\
\text { (USNO B1) }\end{array}$ & 18192006-2045412 & $1-3$ & -0.80 & II & $\ldots$ \\
\hline $24 \mathrm{a}$ & 274.83715 & -20.76199 & $0.05 \pm 0.03$ & $3.31 \pm 0.22$ & $\begin{array}{c}38 \\
(80)\end{array}$ & $\ldots$ & $\begin{array}{l}\text { Empty optical/IR } \\
\text { field }\end{array}$ & $\ldots$ & $\ldots$ & & $\ldots$ & $\ldots$ \\
\hline $24 b$ & 274.83613 & -20.76291 & $0.11 \pm 0.05$ & $0.08 \pm 0.04$ & 1.4 & $\ldots$ & $\ldots$ & 18192066-2045464 & $1-2$ & -0.44 & II & $\ldots$ \\
\hline $24 c$ & 274.83558 & -20.76343 & $0.08 \pm 0.04$ & $0.05 \pm 0.03$ & 0.96 & $\ldots$ & $\ldots$ & $\ldots$ & $1-4$ & 0.76 & Flat & $\ldots$ \\
\hline $24 d$ & 274.83488 & -20.76385 & $0.01 \pm 0.01$ & $0.10 \pm 0.05$ & 1.4 & $\ldots$ & $\ldots$ & $\ldots$ & $\begin{array}{c}1-4 \\
+ \text { MIPS } 24\end{array}$ & 0.45 & Flat & $\ldots$ \\
\hline 25 & 274.83793 & -20.76574 & $0.32 \pm 0.07$ & $0.85 \pm 0.11$ & 13 & $\ldots$ & $\begin{array}{c}\text { HL 11? } \\
0692-0595862 ?\end{array}$ & 18191175-2047264 & $\begin{array}{c}1-4 \\
+ \text { MIPS } 24\end{array}$ & 0.57 & I & $\ldots$ \\
\hline 26 & 274.83737 & -20.76937 & $1.72 \pm 0.15$ & $0.60 \pm 0.09$ & 12 & 6.7 & $\begin{array}{c}\text { CXOPTM J181920.9- } \\
204609 \\
0692-0595859\end{array}$ & 18192095-2046095 & $\begin{array}{c}1-3 \\
+ \text { MIPS } 24\end{array}$ & -0.13 & II & 3 \\
\hline
\end{tabular}

Notes.

${ }^{\text {a }}$ Values in parentheses are unabsorbed luminosities when measured in the $0.3-10 \mathrm{keV}$ band.

${ }^{\mathrm{b}}$ Unresolved combination of sources $21 \& 22$.

${ }^{c}$ SED slope as in Greene et al. (1994).

References: (1) Hartigan \& Lada (1985); (2) Martí et al. (1993); (3) Pravdo et al. (2004). 


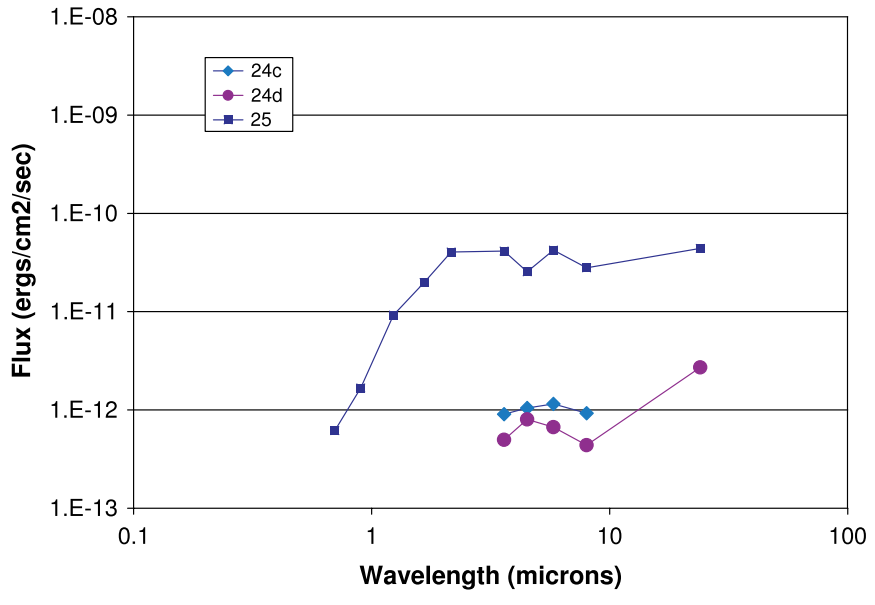

(a)

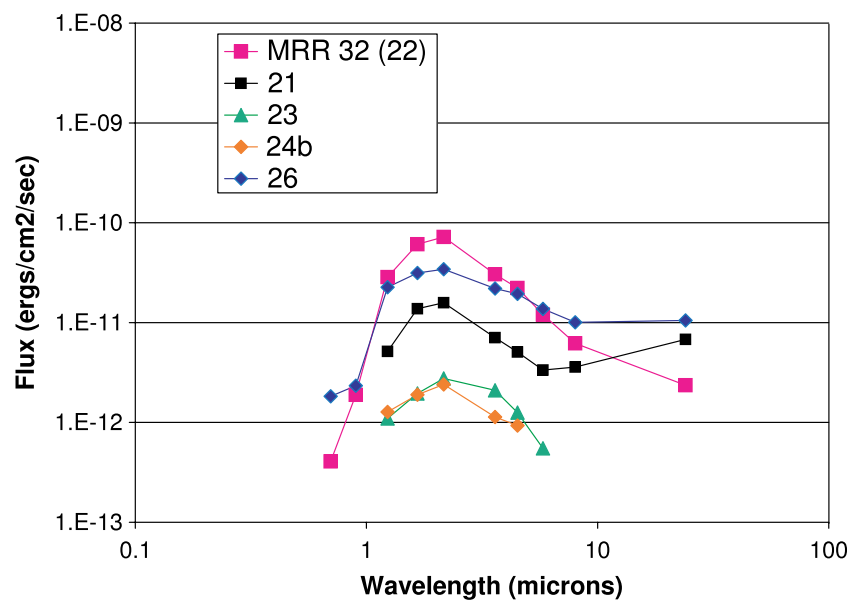

(b)

Figure 9 SEDs of the optical/IR counterparts of the X-ray sources in the MRR 32 cluster: (a) flat, and (b) Class II and III (MRR 32, itself) sources.

(A color version of this figure is available in the online journal.)

other Class I objects, a process described in Ogura et al. (2002). The line of triggered stars has a position angle, P.A. $\sim 125^{\circ}$. This is roughly perpendicular to the molecular outflow axis which has a P.A. $=20 \pm 10$ (Martí et al. 1998). Interestingly, the orientation of the Class I stars that Getman et al. (2007) speculate is the result of triggered star formation, and is also roughly perpendicular to the outflow axis of the most luminous mid-IR star, IRAS $21391+5802$. Perhaps the star formation triggering is damped in the direction of the molecular outflow where the available energy is employed in the outflow kinetics.

Source 15 is the only Class I source that is also detected in soft $\mathrm{X}$-rays. The $N_{H}$ estimated from its X-ray spectrum corresponds to $A_{V} \leqslant 4.5$ (Gorenstein 1975 ), considerably less than for the other Class I objects with measurable values. This may mean that the protostar is on the near side of the molecular cloud and thus suffers less absorption. Sources 24c and 24d in the MRR 32 cluster may be similarly located.

Source 7 is in its own small neighborhood of source confusion. Within $2^{\prime \prime}$ there are sources reported in bands from the $\mathrm{mm}$ source to, now, the X-ray. The sources appear in two groups, one to the northeast and one to the southwest, separated by about 1 1".5. The southwest group contains the X-ray source 7, the optical object HL 34 (Hartigan \& Lada 1985), which possibly equals USNO B1 0692-0595791 (Monet et al. 2003), and the 2.6-mm source, CS 1 (Yamashita et al. 1991). In the northeast are 2MASS 18191175-2047264, IRS 8 (Stecklum et al. 1997), or "object b" (Aspin et al. 1991), and our Spitzer/IRAC detections in bands 3 and 4. Figure 3(a) shows that the SED of the source to the northeast ("IRS") is a Class I protostar with, perhaps, a flaring disk. It may also be two superimposed IR sources as the SED could be a composite spectrum.

Source 7 is a soft X-ray source only, has a luminosity lower by $\sim 10$ in X-rays than the nearby objects, and has a factor of greater than 2 variability between the epochs. The optical source, HL 34, is coincident within 0 '. 2 of source 7 . CS 1 , however, may be offset by $\sim 0^{\prime} .5$ to the southeast. If this is the case, then the YSO hypothesized by Yamashita et al. for CS 1 may be unrelated to the X-ray and optical object. Yamashita et al. derived $N\left(\mathrm{H}_{2}\right)$ $=1 \times 10^{24} \mathrm{~cm}^{-2}$, toward CS 1 , making an associated soft X-ray source and optical source unlikely. Instead we revive the idea that source $7 / \mathrm{HL} 34$ is a foreground star. Summarizing, there may be as many as three sources in this $2^{\prime \prime}$ neighborhood: a Class I source to the northeast (= IRS 8 ), the embedded mm source CS 1, and the X-ray/optical source that may be in the foreground.

\subsection{GGD 27-X}

GGD $27-\mathrm{X}$ is an instance in which we may measure the $\mathrm{X}$-ray spectrum of an optically obscured high-mass protostar with a massive outflow, here a PMS B0 star. The X-rays are coincident with the center of the molecular outflow and the IR emission from GGD 27-ILL (Figure 5) to within the measurement uncertainty. The low-energy X-ray absorption seen in its spectrum corresponds to at least $A_{V} \geqslant 30$ mag (best fit of $\sim 100 \mathrm{mag}$ ), consistent with estimates of the reddening from the IR (e.g., Aspin 1994). These two results contrast with the situation in L1551/IRS 5, wherein the X-rays are offset from the IR emission and the absorption is inconsistent. In that case Bally et al. (2003) conclude that the X-rays arise from the shock created by the outflow.

Table 1 shows the X-ray luminosity of GGD 27-X that includes the effect of the low-energy absorption. If, however, we assume that the absorption is external to the source, then the unabsorbed luminosity is in the range $1.5-12 \times 10^{31} \mathrm{erg} \mathrm{s}^{-1}$ based upon the inferred column density limits. The bolometric luminosity of GGD 27-ILL is estimated at $1.9 \times 10^{4} L_{\odot}$ (McCutcheon et al. 1995). This results in $L_{X} / L_{\mathrm{bol}}=(2-16) \times$ $10^{-7}$. We compare these values with the few prior $X$-ray measurements of other possible high-mass YSOs with outflows shown in Table 3 . The X-ray luminosities are all less than $0.2 \times$ $10^{31} \mathrm{erg} \mathrm{s}^{-1}$, with GG7 27-X potentially the highest by a factor of several. The X-ray temperatures are typically $\geqslant 10^{7} \mathrm{~K}$. The comparison with IRS 1 (NGC 2071), which is also in an UCHII (Snell \& Bally 1986) is the best, while the comparison to the others is strained. IRS 1 and TC1 have $L_{\mathrm{bol}}$ smaller by a factor of $\sim 20$ compared with GGD 27-ILL, and IRS 4 is about a factor of 8-60 less luminous in X-rays than GGD 27-X. Furthermore, IRS 4 drives a "bipolar nebula" (Giardino et al. 2004; see also Bally et al. 1983) that may be the remnant of past outflow activity. This last interpretation is also consistent with its greater age (Table 3). A similarly ambiguous situation exists in TC1 (Cernicharo et al. 1998) where an outflow is not confirmed. The lack of perfect comparisons is due in part to the following reasons: some possible YSOs have X-ray emission but are not classified because they lack IR counterparts (Takagi et al. 2002). In other cases, such as in R Mon, Preibisch et al. (2002) showed that the IRS 3 X-ray sources were unlikely to be the outflow source (see also Kohno et al. 2002). 
Table 3

Comparison of GGD 27-X with Other Possible Young Massive-Star-Outflow X-ray sources

\begin{tabular}{|c|c|c|c|c|c|c|c|c|}
\hline Outflow Source & Region & Spectral Type & $\begin{array}{c}\text { Age } \\
\left(10^{5} \mathrm{Yr}\right)\end{array}$ & $\begin{array}{l}\text { Outflow Velocity } \\
\left(\mathrm{km} \mathrm{s}^{-1}\right)\end{array}$ & $\begin{array}{c}\text { X-ray } \\
T\left(10^{7} \mathrm{~K}\right) \\
\end{array}$ & $L_{\mathrm{X}}\left(10^{31} \mathrm{erg} \mathrm{cm}^{-2} \mathrm{~s}^{-1}\right)$ & $L_{x} / L_{\mathrm{bol}}\left(10^{-7}\right)$ & References \\
\hline GGD 27-ILL & GGD 27 & B0 & $\leqslant 1$ & 700 & $>1$ & $1.5-12$ & $0.7-2$ & $\begin{array}{c}\text { This paper, } \\
\text { Martí et al. (1993) }\end{array}$ \\
\hline IRS 1 & NGC 2071 & B0-B5 & $\leqslant 1$ & 70 & $0.2-1$ & 1.5 & $>50$ & Skinner et al. (2007) \\
\hline $\mathrm{TC} 1$ & Trifid Nebula & $\ldots$ & $<3$ & $\ldots$ & 1 & 1.9 & 80 & $\begin{array}{l}\text { Rho et al. (2004, 2006), } \\
\text { Cernicharo et al. (1998) }\end{array}$ \\
\hline IRS 4 & S106 & O7-B0 & $10-20$ & 100 & $1-3$ & 0.2 & $0.05-0.1$ & $\begin{array}{l}\text { Giardino et al. (2004), } \\
\text { Simon et al. (1983) }\end{array}$ \\
\hline
\end{tabular}

Prior observations of young, unobscured $\mathrm{B} 0$ and $\mathrm{B} 0.5$ stars in X-rays show X-ray luminosities, $6 \times 10^{28}-2 \times 10^{31} \mathrm{erg} \mathrm{s}^{-1}$, that are consistent at their high end with the minimum luminosity of GGD 27 (e.g., Broos et al. 2007). The GGD 27 X-ray luminosity is also consistent with BM Ori, a PMS B0.5 star in the Orion Nebula Cluster (ONC), but at the high end of ONC stars with spectral type earlier than B2 (Grosso et al. 2005). The GGD $27-\mathrm{X}$ temperature of $\geqslant 10^{7} \mathrm{~K}$ is consistent with prior measurements of B stars and massive-star-outflow sources (Table 3), but higher than that seen from HH shocks (e.g. PTM).

The factor $\geqslant 10$ variability of GGD $27-\mathrm{X}$ between the two epochs also suggests that the X-rays originate in the star rather than the circumstellar outflow. In contrast with the X-rays, the radio luminosity of the outflow remains nearly constant, even though its detailed morphology changes on a timescale of years (Martí et al. 1998). Possible variable X-ray emission mechanisms from a massive star include changes in its stellar wind, an unseen companion, and other magnetic processes. The stellar wind hypothesis, for example, implies a shock velocity $v_{s} \geqslant\left(16 k T / 3 \mu m_{p}\right)^{\frac{1}{2}}=670 \mathrm{~km} \mathrm{~s}^{-1}$, where $T$ is the observed $\mathrm{X}$-ray lower temperature limit, $m_{p}$ is the proton mass, and $\mu$ is the mean molecular mass in the wind (e.g., Pravdo et al. 2001). This and higher velocities are attainable in B-star stellar winds (Cassinelli et al. 1994). Since the X-ray temperatures are similar for all the outflow sources listed in Table 3, their required shock velocities would be about the same as our estimate for GGD 27. It is interesting to note, however, that only in GGD 27 does the outflow velocity reach this value.

\subsection{Comparing the Two Clusters and a Blank-Field Variable Source in Each}

Like the GGD 27 cluster, the MRR 32 cluster may be contained within the giant molecular cloud discovered by Saito et al. (1999). However, for the object MRR 32 itself, see Section 4.4. If so, it is within the volume of space influenced by GGD 27ILL, since the latter is thought to have powered the HH 80 North outflow, several arcsec further northeast (Martí et al. 1993). The distribution of identified Class types is later than that near GGD 27-ILL, split about equally between flat and Class II sources with the brightest source, MRR 32 itself, the only Class III object. The enhanced $24 \mu \mathrm{m}$ emission suggests that the Class II objects are classical T Tauri stars with disks (e.g., Scholz et al. 2007).

Both clusters contain sources, 11 in GGD 27 and 24a in MRR 32, that are very luminous in X-rays, $L_{X} \geqslant 10^{32} \mathrm{erg} \mathrm{s}^{-1}$, without optical/IR/radio counterparts, and that brightened by a factor of $\geqslant 6$ in the 4 years between observations. Both sources exhibit probable shorter-term flaring behavior by a factor of a few over timescales of tens of thousands of seconds (Figures 10(a) and 10(b)). Kolmogorov-Smirnov tests for constancy showed a $6.2 \%$ probability for source 11 and $30 \%$ probability for source 24a. For source 11, the low-energy absorption measured in its X-ray spectrum corresponds to $A_{V}=52(+56$, $-32) \mathrm{mag}$. The unabsorbed luminosity is $1 \times 10^{32} \mathrm{erg} \mathrm{s}^{-1}$, high for a PMS star. In the ONC, for example, only $\Theta^{1} \mathrm{C}$, an $\mathrm{O} 6 \mathrm{star}$ at $\sim 10^{33} \mathrm{erg} \mathrm{s}^{-1}$, is more luminous. If source 11 is an intermediate-mass star with $L_{x} / L_{\text {bol }}$ typically less than $2 \times 10^{-4}$, it would have $L_{\mathrm{bol}} \geqslant 10^{2} L_{\odot}, \geqslant 1 \%$ of the luminosity of GGD 27-ILL. Similarly 24 a has an unabsorbed X-ray luminosity of $8 \times 10^{31} \mathrm{erg} \mathrm{s}^{-1}$. Such blank-field sources are not unprecedented. Prisinzano et al. (2007) list 7 similar sources in the Orion Molecular Cloud. All have $N_{H} \geqslant 10^{23} \mathrm{H} \mathrm{cm}^{-2}$, but the highest inferred $L_{X}$ (without absorption) is $3 \times 10^{30} \mathrm{erg} \mathrm{s}^{-1}$ (Grosso et al. 2005) — 30 times less luminous than these in the Saito cloud.

Both sources appear at the end of a linear string of $\mathrm{X}$-ray/Class I or flat objects. Source 24a has a projected separation of only $\sim 4000 \mathrm{AU}$ (cf. Winston et al. 2007) from the Class II, X-ray object, 24b, which is only moderately absorbed based upon its ratio of soft to hard X-ray counts. Source 24a has an equivalent $A_{V}=32 \mathrm{mag}$. These objects may be protostars that have only now emerged in high-density regions during a more recent episode of star formation. These high-density regions would be created during past episodes on the boundaries of the star forming regions. The orientation of the string in MRR 32 is at P.A. $\sim 45^{\circ}$, closer to the GGD 27 outflow axis. Both of the blank-field sources lie downstream from their linear grouping. Teixeira et al. (2006) also discuss linear configurations of protostars and conclude that the spacing of the stars in NGC 2264 is comparable to the Jeans length in that medium. For GGD 27 a higher temperature and lower density (McCutcheon et al. 1995) make the Jeans length considerably higher than the separation in either string.

A less speculative interpretation for the blank-field sources is that they are "hidden" Class III objects, i.e., obscured by intervening material. This would be consistent with the large $\mathrm{X}$-ray luminosities, variability, and the lack of evidence for any IR emission.

\subsection{MRR 32}

We associate the X-ray source 22 with the radio continuum, optical, and IR source MRR 32. It has $L_{X}=3-6 \times 10^{31} \mathrm{erg} \mathrm{s}^{-1}$ if it is within the Saito cloud at $1.7 \mathrm{kpc}$. The optical/IR SED shown in Figure 9(b) is Class III yielding a possible identification as a weak-lined T Tauri star. Its X-ray to bolometric luminosity ratio would be $\sim 10^{-3}$, near saturation, in this case.

As noted in Section 2.1, it is statistically unlikely that any of our sources is extragalactic. However, we cannot rule out the possibility that some, including MRR 32, are foreground objects. Its SED can be modeled in terms of a reddened 


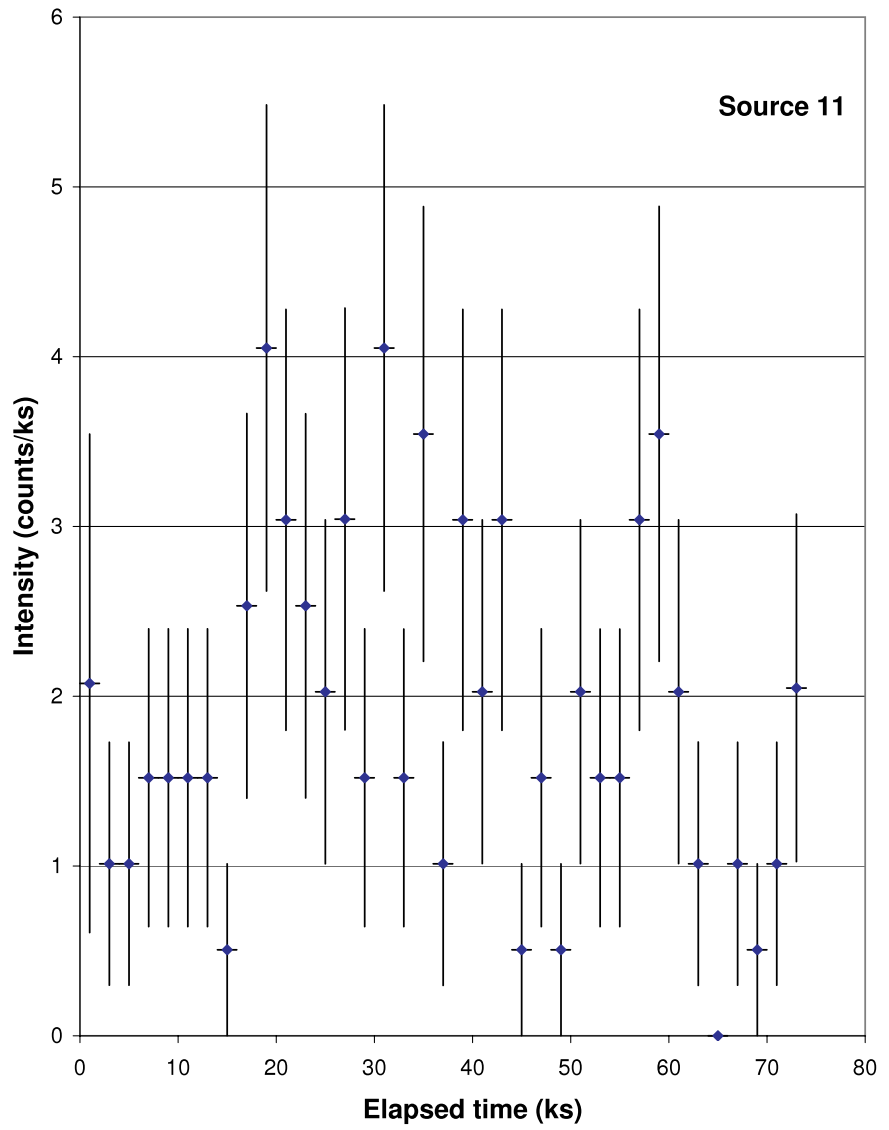

(a)



(b)

Figure 10. X-ray light curves in $2000 \mathrm{~s}$ bins: (a) source 11, (b) source 24a.

(A color version of this figure is available in the online journal.)

foreground low-mass star with modest IR excess. There is, however, no evidence of proper motion in this object based upon our optical astrometry at epochs spanning $\sim 50$ years. Still an intermediate distance of $\sim 300 \mathrm{pc}$ would be consistent with the lack of observed proper motion and an X-ray luminosity of $\sim 10^{30} \mathrm{erg} \mathrm{s}^{-1}$, at the high end of the luminosity distribution for low-mass T Tauri stars.

\section{CONCLUSIONS}

GGD 27 is confirmed as a cluster of protostars and PMS stars. We detect X-rays from GGD 27-X = GGD 27-ILL, the high-mass Class I protostar that is the power source of the bipolar outflow. Few such objects have been reported as $\mathrm{X}$-ray sources. Its X-ray properties are consistent with those of stars of its type in other star formation regions. We identified a group of at least three other Class I protostars that are within 0.1 pc of GGD 27-ILL. X-ray variability over timescales of years by a factor $\geqslant 10$ is typical. We also identify the nearby MRR 32 cluster. Both the GGD 27 and MRR 32 clusters contain a blankfield optical/IR/radio source with similar X-ray propertieshigh luminosity, variability, and hard, absorbed spectra-that may be representatives of a newer generation of protostars.

The research described in this paper was performed in part by the Jet Propulsion Laboratory, California Institute of Technology, under contract with the National Aeronautics and Space Administration. We thank K. Getman, A. Burrows, Y. Maeda, \& M. Tamura for helpful discussions. This research has made use of the NASA/IPAC Infrared Science Archive including the Spitzer archive, which is operated by the Jet Propulsion Laboratory, California Institute of Technology, under contract with the National Aeronautics and Space Administration. This research has made use of the SIMBAD database, operated at CDS, Strasbourg, France. This publication makes use of data products from the Two Micron All Sky Survey, which is a joint project of the University of Massachusetts and the Infrared Processing and Analysis Center/California Institute of Technology, funded by the National Aeronautics and Space Administration and the National Science Foundation. This research was supported by NASA contract NAS 8-01128. Government sponsorship acknowledged. Y.T. acknowledges support from the Grants-in-Aid for Scientific Research (number 20540237) by the Ministry of Education, Culture, Sports, Science and Technology. Copyright 2008 California Institute of Technology.

\section{REFERENCES}

Aspin, C. 1994, A\&A, 281, L29

Aspin, C., \& Geballe, T. R. 1992, A\&A, 266, 219

Aspin, C., McCaughrean, M. J., Casali, M. M., \& Geballe, T. R. 1991, A\&A, 252,299

Aspin, C., et al. 1994, A\&A, 292, L9A

Backus, C. R., Velusamy, T., Thompson, T. J., \& Arballo, J. K. 2005, ASASS XIV, ASP Conf. Series 347, ed. P. L. Shopbell, M.C. Britton, \& R. Ebert (San Francisco, CA: ASP), 61

Bally, J., Feigelson, E., \& Reipurth, B. 2003, ApJ, 584, 843

Bally, J., Snell, R. L., \& Predmore, R. 1983, ApJ, 272, 154 
Bertin, E., \& Amouts, S. 1996, A\&Ap Suppl., 117, 393

Bica, E., Dutra, C. M., \& Barbuy, B. 2003, A\&A, 397, 177

Broos, P. S., Feigelson, E. D., Townsley, L. K., Getman, K. V., Wang, J., Garmire, G. P., Jiang, Z., \& Tsuboi, Y. 2007, ApJS, 169, 353

Cassinelli, J. P., Cohen, D. H., MacFarlane, J. J., Sanders, W. T., \& Welsh, B. Y. 1994, ApJ, 421, 705

Cernicharo, J., et al. 1998, Science, 282, 462

Fazio, G. G., et al. 2004, ApJS, 154, 10

Feigelson, E. D., \& Townsley, L. K. 2008, ApJ, 673, 354

Feigelson, E. D., et al. 2002, ApJ, 574, 258

Freeman, P. E., Kashyap, V., Rosner, R., \& Lamb, D. Q. 2002, ApJS, 138, 185

Getman, K., Feigelson, E. D., Garmire, G., Broos, P., \& Wang, Junfeng 2007, ApJ, 654, 316

Ghavamian, P., \& Hartigan, P. 1998, ApJ, 501, 687

Giardino, G., Favata, F., \& Micela, 2004, A\&A, 424, 965

Gómez, Y., Rodríguez, L. F., \& Martí, J. 1995, ApJ, 453, 268

Gorenstein, P. 1975, ApJ, 198, 95

Greene, T. P., Wilking, B. A., André, P., Young, E. T., \& Lada, C. J. 1994, ApJ, 434,614

Grosso, N., et al. 2005, ApJS, 160, 530

Gyulgudaghian, A. L., Glushkov, , Yu, I., \& Denisyuk, E. K. 1978, ApJ, 224, L137

Hartigan, P., \& Lada, C. J. 1985, ApJS, 59, 383

IRAS Point Source Catalog 1985, Joint IRAS Science Working Group (Washington, D.C.: U.S. Government Printing Office)

Kohno, M., Koyama, K., \& Hamaguchi, K. 2002, ApJ, 567, 423

Kurtz, S., Churchwell, E., \& Wood, D. O. S. 1994, ApJS, 91, 659

Lampton, M., Margon, B., \& Bowyer, S. 1976, ApJ, 208, 177

Makovoz, D., \& Marleau, F. R. 2005, PASP, 117, 1113

Martí, J., Rodríguez, L., \& Reipurth, B. 1993, ApJ, 416, 208

Martí, J., Rodríguez, L., \& Reipurth, B. 1995, ApJ, 449, 184

Martí, J., Rodríguez, L., \& Reipurth, B. 1998, ApJ, 502, 337

McCutcheon, W. H., Dewdney, P. E., Purton, C. R., \& Sato, T. 1991, AJ, 101, 1435

McCutcheon, W. H., Sato, T., Purton, C. R., Matthews, H. E., \& Dewdney, P. E. 1995, AJ, 110, 1762

Mewe, R., Gronenschild, E. B. H. M., \& van den Oord, G. H. J. 1985, A\&A, 62, 197

Monet, D., et al. 2003, AJ, 125, 984

Ogura, K., Sugitani, K., \& Pickles, A. 2002, AJ, 123, 2597

Panagia, N. 1973, AJ, 78, 929
Pravdo, S. H., Feigelson, E. D., Garmire, G., Maeda, Y., Tsuboi, Y., \& Bally, J. 2001, Nature, 413, 708

Pravdo, S. H., Tsuboi, Y., \& Maeda, Y. 2004, ApJ, 605, 259 (PTM)

Preibisch, T., Balega, Y. Y., Schertl, D., \& Weigelt, G. 2002, A\&A, 392, 945

Prisinzano, L., et al. 2007, ApJ, 677, 401

Prisinzano, L., et al. 2008, ApJ, 677, 401

Reipurth, B., \& Graham, J. A. 1988, A\&A, 202, 219

Rho, J, Ramirez, S. V., Corcoran, M. F., Hamaguchi, K., \& Lefloch, 2004, ApJ, 607, 904

Rho, J., Reach, W. T., Lefloch, B., \& Fazio, G. G. 2006, ApJ, 643, 965

Rieke, G. H., et al. 2004, ApJS, 154, 25

Rodríguez, L. F., Moran, J. M., Ho, P. T. P., \& Gottlieb, E. W. 1980, ApJ, 235, 845

Rodríguez, L. F., \& Reipurth, B. 1989, Rev. Mex. AA, 17, 59

Saito, H., et al. 1999, PASJ, 51, 819

Scholz, A., Jayawardhana, R., Wood, K., Meeus, G., Stelzer, B., Walker, C., \& O'Sullivan, M 2007, ApJ, 660, 1517

Simon, M., Felli, M., Cassar, L., Fischer, J., \& Massi, M. 1983, ApJ, 266, 623

Skinner, S. L., Simmons, A. E., Audard, M., \& Güdel, M. 2007, ApJ, 658, 1144

Skrutskie, M. F., et al. 2006, AJ, 131, 1163

Snell, R. L., \& Bally, J. 1986, ApJ, 303, 683

Stecklum, B., Feldt, M., Richchi, A., Calamai, G., \& Lagage, P. O. 1997, ApJ, 479,339

Sugitani, K., Tamura, M., \& Ogura, K. 1995, ApJ, 455, L39

Takagi, S., Murakami, H., \& Koyama, K. 2002, ApJ, 573, 275

Teixeira, P. S., et al. 2006, ApJ, 636, L45

Thompson, M. A., Hatchell Walsh, J. A. J., MacDonald, G. H., \& Millar, T. J. 2006, A\&A, 453, 1003

Velusamy, T., Langer, W. D., \& Marsh, K. A. 2007, ApJ, 668, L159

Weisskopf, M. C., Brinkman, B., Canizares, C., Garmire, G., Murray, S., \& Van Speybroeck, L. P. 2002, PASP, 114,

Werner, M. W., et al. 2004, ApJS, 154, 1

Winston, E., et al. 2007, ApJ, 669, 493

Wolk, S. J., Spitzbart, B. D., Bourke, T., \& Alves, 2006, AJ, 132, 1100

Yamashita, T., Hayashi, S. S., Kaifu, N., Kameya, O., Ukita, N., \& Hasegawa, T. 1989, ApJ, 347, 894

Yamashita, T., Murata, Y., Kawabe, R., Kaifu, N., \& Tamura, M. 1991, ApJ, 33, 560

Yamashita, T., Sato, S., Nagata, T., Suzuki Hough, H. J. H., McLean, I. S., Garden, R., \& Gatley, I. 1987, A\&A, 177, 258

Yorke, H. W. 1986, ARAA, 24, 49 